Tom 25 № 4

\title{
ЛИНЕЙНОЕ ВОССТАНОВЛЕНИЕ ПСЕВДОДИФФЕРЕНЦИАЛЬНЫХ ОПЕРАТОРОВ НА КЛАССАХ ГЛАДКИХ ФУНКЦИЙ НА $m$-МЕРНОМ ТОРЕ. $\mathrm{II}^{1}$
}

\author{
Д. Б. Базарханов
}

\begin{abstract}
В предлагаемой работе формулируется и обсуждается задача оптимального восстановления значений псевдодифференциальных операторов $T_{a}$ на $m$-мерном торе $\mathbb{T}^{m}$ с символами $a$ из классов $\widetilde{\Psi}_{\epsilon \theta}^{\tau \mathrm{m}}[v ; \mathrm{K}, \mathrm{L}]$, на распределениях $f$ из классов $\mathrm{B}_{p q}^{s \mathrm{~m}}\left(\mathbb{T}^{m}\right)$ типа Никольского - Бесова и $\mathrm{L}_{p q}^{s \mathrm{~m}}\left(\mathbb{T}^{m}\right)$ типа Лизоркина - Трибеля по конечной спектральной информации о символе оператора и о распределении (конечные наборы коэффициентов Фурье символа оператора и распределения). Доказывается, что оптимальным (или, по крайней мере, линейным оптимальным) по порядку методом восстановления в этой задаче для ряда соотношений между параметрами класса символов, класса распределений и объемлющего пространства является метод $\Upsilon_{\Lambda(\gamma, N)}$, построенный и изученный в части I данной работы автора $(2018)$; при этом величина (линейного) оптимального восстановления имеет точный порядок соответствующего поперечника Фурье классов $\mathrm{B}_{p q}^{s-\tau \mathrm{m}}\left(\mathbb{T}^{m}\right)$ и $\mathrm{L}_{p q}^{s-\tau \mathrm{m}}\left(\mathbb{T}^{m}\right)$ соответственно (теорема 1$)$. Попутно утверждение теоремы 1 части I доказывается при "естественных" условиях на дифференциальные параметры $\tau$ классов символов $\widetilde{\Psi}_{\epsilon \theta}^{\tau \mathrm{m}}[v ; \mathrm{K}, \mathrm{L}]$ и $s$ пространств $B_{p q}^{s \mathrm{~m}}\left(\mathbb{T}^{m}\right)$ типа Никольского - Бесова и $L_{p q}^{s \mathrm{~m}}\left(\mathbb{T}^{m}\right)$ типа Лизоркина - Трибеля; кроме того, устанавливается, что оценки сверху из теоремы 1 на самом деле являются точными в смысле порядка (см. теорему 3).
\end{abstract}

Ключевые слова: псевдодифференциальный оператор на $m$-мерном торе, класс символов (типа произведения), пространство распределений типа Никольского - Бесова / Лизоркина - Трибеля, оптимальное восстановление класса операторов, оценки погрешности оптимального восстановления, поперечник Фурье.

D. B. Bazarkhanov. Linear recovery of pseudodifferential operators on classes of smooth functions on an $m$-dimensional torus. II.

We formulate and discuss a problem of optimal recovery of values $T_{a} f$ of pseudodifferential operators $T_{a}$ on an $m$-dimensional torus $\mathbb{T}^{m}$ with symbols $a$ from the classes $\Psi_{\epsilon \theta}^{\tau \mathrm{m}}[v ; \mathrm{K}, \mathrm{L}]$ on distributions $f$ from the classes $\mathrm{B}_{p q}^{s \mathrm{~m}}\left(\mathbb{T}^{m}\right)$ of Nikol'skii-Besov type and $\mathrm{L}_{p q}^{s \mathrm{~m}}\left(\mathbb{T}^{m}\right)$ of Lizorkin-Triebel type from finite spectral information about the symbol of the operator and the distribution (finite sets of Fourier coefficients of the symbol and the distribution). We show that the recovery method $\Upsilon_{\Lambda(\gamma, N)}$ constructed and studied in 2018 in the first part of this research is order-optimal (or at least linear order-optimal) in this problem for a number of relations between the parameters of the symbol class, the class of distributions, and the ambient space. Furthermore, the (linear) optimal recovery error has exact order of the corresponding Fourier widths of the classes $\mathrm{B}_{p q}^{s-\tau \mathrm{m}}\left(\mathbb{T}^{m}\right)$ and $\mathrm{L}_{p q}^{s-\tau \mathrm{m}}\left(\mathbb{T}^{m}\right)$, respectively (Theorem 1). Simultaneously, the claim of Theorem 1 from part I of this research is proved under "natural" conditions on the differential parameters $\tau$ of the symbol classes $\widetilde{\Psi}_{\epsilon \theta}^{\tau \mathrm{m}}[v ; \mathrm{K}, \mathrm{L}]$ and $s$ of the spaces $B_{p q}^{s \mathrm{~m}}\left(\mathbb{T}^{m}\right)$ of Nikol'skii-Besov type and $L_{p q}^{s \mathrm{~m}}\left(\mathbb{T}^{m}\right)$ of Lizorkin-Triebel type. It is also established that the upper estimates in Theorem 1 are order-exact (see Theorem 3).

Keywords: pseudodifferential operator on an $m$-dimensional torus, class of symbols (of product type), Nikol'skii-Besov / Lizorkin-Triebel space of distributions, optimal recovery of an operator class, error bounds of optimal recovery, Fourier width.

MSC: 41A45, 42B05, 35S05, 58J40

DOI: $10.21538 / 0134-4889-2019-25-4-15-30$

Посвящается Виталию Ивановичу Бердыщеву в связи с его восвмидесятилетием

\section{1. Задача оптимального восстановления класса операторов}

Теория оптимального восстановления изучает методы приближенного восстановления (значений) различных операторов (на классах элементов), построенные на основе некоторой (вообще говоря, неполной) информации о самих операторах и/или об элементах, на которые они

\footnotetext{
${ }^{1}$ Работа выполнена при поддержке гранта АР05133257 МОН РК.
} 
действуют, оптимальные в том или ином смысле. Различным конкретным задачам, конкретным методам восстановления, общим вопросам оптимального восстановления посвящено много работ (см., например, работы [1-6] и библиографию в них).

Здесь мы обсудим известную задачу оптимального восстановления (индивидуального) оператора по семейству операторов информации об элементах, на которые он действует, и сформулируем вариант задачи оптимального восстановления класса операторов по двум семействам операторов информации (одно - об операторах, значения которых предполагается восстанавливать, другое - об элементах), который будет исследован здесь для класса псевдодифференциальных операторов, рассмотренного в части I (Базарханов Д. Б. Линейное восстановление псевдодифференциальных операторов на классах гладких функций на $m$-мерном торе. I // Тр. Ин-та математики и механики УрО РАН. 2018. Т. 24, № 4. С. 57-79) данной работы. Кроме того, разберем элементарный пример задачи первого типа, который демонстрирует, что и в этом случае было бы вполне естественно рассматривать/привлечь операторы (неполной) информации об операторе, который требуется восстановить.

Предположим, что заданы множество $\mathrm{F}$, линейное нормированное пространство $Y$ с нормой $\|\cdot \mid Y\|$ и оператор $T$, отображающий $\mathrm{F}$ в $Y$. Пусть еще имеется оператор информации $\mathcal{I}$, действующий из $\mathrm{F}$ в линейное пространство $Z_{\mathcal{I}}\left(\mathcal{I}: \mathrm{F} \rightarrow Z_{\mathcal{I}}: f \mapsto \mathcal{I}(f)\right)$.

Задачу оптимального восстановления оператора $T$ на множестве $\mathrm{F}$ по информации $\mathcal{I}$ об элементах $f$ из $\mathrm{F}$ сформулируем в следующем (достаточном для наших целей) виде (подробнее см., например, $[1-4 ; 6]$.

Будем считать, что информация неполна, т. е. что оператор $\mathcal{I}$ не является инъективным отображением. Произвольное отображение $\Upsilon: \mathcal{I}(\mathrm{F}) \rightarrow Y$ называется методом восстановления, а величина

$$
\mathfrak{R}(T, \mathrm{~F} ; \mathcal{I}, \Upsilon ; Y):=\sup \{\|T f-\Upsilon(\mathcal{I}(f))|Y \|| f \in \mathrm{F}\}
$$

- погрешностью метода $\Upsilon$ на множестве F. Требуется вычислить (или, по крайней мере, оценить) погрешность

$$
\mathfrak{S}(T, \mathrm{~F} ; \mathcal{I} ; Y):=\inf \{\mathfrak{R}(T, \mathrm{~F} ; \mathcal{I}, \Upsilon ; Y) \mid \Upsilon\}
$$

оптимального восстановления (значений) оператора $T$ (в пространстве $Y$ ) на множестве $\mathrm{F}$ по информации $\mathcal{I}$, а также построить метод восстановления $\Upsilon^{\circ}$, называемый оптимальным (если таковой существует), на котором достигается нижняя грань в (1.1), или, по крайней мере, метод $\Upsilon^{a}$, погрешность которого "близка" к (1.1) в том или ином смысле.

Хорошо известно, что погрешность (1.1) оптимального восстановления выражается в терминах чебышевских радиусов множеств $T\left(\mathcal{I}^{-1}(z)\right)$, где $\mathcal{I}^{-1}(z)=\{f \in \mathrm{F}: \mathcal{I}(f)=z\}$ - прообразы элементов $z \in \mathcal{I}(\mathrm{F})$.

Напомним, что чебышевским радиусом множества $G \subset Y$ называется число

$$
r(G)=\inf \{\sup \{\|w-y|Y \|| y \in G\} \mid w \in Y\}
$$

при этом элемент $c(G) \in Y$, для которого $\sup \{\|c(G)-y|Y \|| y \in G\}=r(G)$ (если таковой существует), называется чебышевским центром множества $G$.

Имеет место равенство (см., например, [4, теорема 1.1] или [6, следствие 1, с. 23])

$$
\mathfrak{S}(T, \mathrm{~F} ; \mathcal{I} ; Y)=\sup \left\{r\left(T\left(\mathcal{I}^{-1}(z)\right)\right) \mid z \in \mathcal{I}(\mathrm{F})\right\}
$$

Кроме того, если для каждого $z \in \mathcal{I}(\mathrm{F})$ множество $T\left(\mathcal{I}^{-1}(z)\right)$ имеет чебышевский центр, то отображение

$$
\Upsilon^{o}: \mathcal{I}(\mathrm{F}) \rightarrow Y: z \mapsto c\left(T\left(\mathcal{I}^{-1}(z)\right)\right)
$$

- оптимальный (вообще говоря, нелинейный) метод восстановления оператора $T$ на множестве $\mathrm{F}$ по информации $\mathcal{I}$ (метод восстановления $\Upsilon^{o}$ еще называют центральным алгоритмом, см., например, [3, гл. 1]). 
Множество $G \subset Y$ называется иентрально-симметричным, если найдется такая точка $y_{0} \in Y$, называемая центром симметрии ( $\Rightarrow G-y_{0}$-симметрично), что $\forall y \in G$ имеем $2 y_{0}-y \in G$.

Теперь дополнительно предположим, что $T$ - линейный оператор, действующий из линейного пространства $X$, объемлющего множество $\mathrm{F}$, в $Y$ и для любого $z \in \mathcal{I}(\mathrm{F})$ множество $\mathcal{I}^{-1}(z)$ центрально-симметрично (с центром симметрии $\left.x(z) \in X\right)$. Тогда, очевидно, множество $T\left(\mathcal{I}^{-1}(z)\right)$ будет $T(x(z))$-симметричным в $Y$. Поскольку центр симметрии центральносимметричного множества в линейном нормированном пространстве является чебышевским центром этого множества (см., например, [6, лемма 3, с. 19]), то отсюда вытекает, что оптимальный метод восстановления оператора $T$ существует и является центральным алгоритмом,

$$
\Upsilon^{o}: \mathcal{I}(\mathrm{F}) \rightarrow Y: z \mapsto T(x(z)) .
$$

В случае, когда вместо одного задано целое семейство операторов информации $\mathfrak{I}=\{\mathcal{I}\}$, естественно рассмотреть следующую задачу оптимального восстановления: найти (или оценить) величину

$$
\mathfrak{S}(T, \mathrm{~F} ; \mathfrak{I} ; Y):=\inf \{\mathfrak{S}(T, \mathrm{~F} ; \mathcal{I} ; Y) \mid \mathcal{I} \in \mathfrak{I}\}
$$

а также оператор информации $\mathcal{I}^{o} \in \mathfrak{I}$ и метод восстановления $\Upsilon^{o}: \mathcal{I}^{o}(\mathrm{~F}) \rightarrow Y$ (если таковые существуют), для которых $\mathfrak{R}\left(T, \mathrm{~F} ; \mathcal{I}^{o}, \Upsilon^{o} ; Y\right)=\mathfrak{S}(T, \mathrm{~F} ; \mathfrak{I} ; Y)$, или хотя бы оператор информации $\mathcal{I}^{a} \in \mathfrak{I}$ и метод восстановления $\Upsilon^{a}: \mathcal{I}^{a}(\mathrm{~F}) \rightarrow Y$, для которых величина $\mathfrak{R}\left(T, \mathrm{~F} ; \mathcal{I}^{a}, \Upsilon^{a} ; Y\right)$ близка к $\mathfrak{S}(T, \mathrm{~F} ; \mathfrak{I} ; Y)$ в том или ином смысле. Подробнее об этих и других задачах оптимального восстановления см., например, [1;2; 3, гл.1; 6 , гл.1].

Переходим к постановке задачи восстановления "класса" операторов на некотором множестве по семействам операторов информации как об операторах, так и об элементах, на которые они действуют.

Пусть $\mathfrak{H}$ - некоторая совокупность (класс) операторов $T$, действующих из множества $\mathrm{F}$ в пространство $Y$, и, как и выше, $\mathfrak{I}=\{\mathcal{I}\}-$ семейство операторов информации об элементах из $\mathrm{F}$, а $\mathfrak{J}=\{\mathcal{J}\}-$ семейство операторов информации об операторах из $\mathfrak{H}$, т. е. операторов $\mathcal{J}$, действующих из $\mathfrak{H}$ в линейные пространства $Z_{\mathcal{J}}\left(\mathcal{J}: \mathfrak{H} \rightarrow Z_{\mathcal{J}}: T \mapsto \mathcal{J}(T)\right.$ ).

Произвольное отображение $\Upsilon: \mathcal{I}(\mathrm{F}) \times \mathcal{J}(\mathfrak{H}) \rightarrow Y$ называется методом восстановления, а величина

$$
\mathfrak{R}(\mathfrak{H}, \mathrm{F} ; \mathcal{I}, \mathcal{J}, \Upsilon ; Y):=\sup \{\|T f-\Upsilon(\mathcal{I}(f), \mathcal{J}(T))|Y \|| f \in \mathrm{F}, T \in \mathfrak{H}\}
$$

- погрешностью метода $\Upsilon$ восстановления класса $\mathfrak{H}$ на множестве $\mathrm{F}$.

Задача оптимального восстановления (значений) операторов $T$ из класса $\mathfrak{H}$ на (элементах $f$ из F) по семействам (неполной) информации $\mathfrak{I}$ об $f$ и $\mathfrak{J}$ о $T$ состоит в следующем: найти (или оценить) величину

$$
\mathfrak{S}(\mathfrak{H}, \mathrm{F} ; \mathfrak{I}, \mathfrak{J} ; Y):=\inf \{\mathfrak{R}(\mathfrak{H}, \mathrm{F} ; \mathcal{I}, \mathcal{J}, \Upsilon ; Y) \mid \Upsilon, \mathcal{I} \in \mathfrak{I}, \mathcal{J} \in \mathfrak{J}\}
$$

а также операторы информации $\mathcal{I}^{o} \in \mathfrak{I}, \mathcal{J}^{o} \in \mathfrak{J}$ и метод восстановления $\Upsilon^{o}: \mathcal{I}^{o}(\mathrm{~F}) \times \mathcal{J}^{o}(\mathfrak{H}) \rightarrow$ $Y$ (если таковые существуют), для которых $\mathfrak{R}\left(\mathfrak{H}, \mathrm{F} ; \mathcal{I}^{o}, \mathcal{J}^{o}, \Upsilon^{o} ; Y\right)=\mathfrak{S}(\mathfrak{H}, \mathrm{F} ; \mathfrak{I}, \mathfrak{I} ; Y)$, или хотя бы операторы информации $\mathcal{I}^{a} \in \mathfrak{I}, \mathcal{J}^{a} \in \mathfrak{J}$ и метод восстановления $\Upsilon^{a}: \mathcal{I}^{a}(\mathrm{~F}) \times \mathcal{J}^{a}(\mathfrak{H}) \rightarrow Y$, для которых величина $\mathfrak{R}\left(\mathfrak{H}, \mathrm{F} ; \mathcal{I}^{a}, \mathcal{J}^{a}, \Upsilon^{a} ; Y\right)$ близка к $\mathfrak{S}(\mathfrak{H}, \mathrm{F} ; \mathfrak{I}, \mathfrak{J} ; Y)$ в том или ином смысле.

Метод восстановления $\Upsilon^{\prime}: \mathcal{I}(\mathrm{F}) \times \mathcal{J}(\mathfrak{H}) \rightarrow Y$ будем называть линейным, если отображение $\Upsilon^{\prime}(\mathcal{I}(f), \mathcal{J}(T))$ линейно по $\mathcal{I}(f)$ при фиксированном $\mathcal{J}(T)$.

Задача линейного оптимального восстановления класса операторов $\mathfrak{H}$ на множестве $\mathrm{F}$ по семействам информации $\mathfrak{I}$ и $\mathfrak{J}$ формулируется аналогично: найти (или оценить) величину

$$
\mathfrak{S}^{\prime}(\mathfrak{H}, \mathrm{F} ; \mathfrak{I}, \mathfrak{J} ; Y):=\inf \left\{\mathfrak{R}\left(\mathfrak{H}, \mathrm{F} ; \mathcal{I}, \mathcal{J}, \Upsilon^{\prime} ; Y\right) \mid \Upsilon^{\prime}, \mathcal{I} \in \mathfrak{I}, \mathcal{J} \in \mathfrak{J}\right\}
$$

а также операторы информации $\mathcal{I}^{o} \in \mathfrak{I}, \mathcal{J}^{o} \in \mathfrak{J}$ и линейный метод восстановления $\Upsilon^{\prime o}: \mathcal{I}^{o}(\mathrm{~F}) \times$ $\mathcal{J}^{o}(\mathfrak{H}) \rightarrow Y$ (если таковые существуют), для которых $\mathfrak{R}\left(\mathfrak{H}, \mathrm{F} ; \mathcal{I}^{o}, \mathcal{J}^{o}, \Upsilon^{\prime o} ; Y\right)=\mathfrak{S}^{\prime}(\mathfrak{H}, \mathrm{F} ; \mathfrak{I}, \mathfrak{I} ; Y)$, 
или хотя бы операторы информации $\mathcal{I}^{a} \in \mathfrak{I}, \mathcal{J}^{a} \in \mathfrak{J}$ и линейный метод восстановления $\mathfrak{\Upsilon}^{\prime a}:$ $\mathcal{I}^{a}(\mathrm{~F}) \times \mathcal{J}^{a}(\mathfrak{H}) \rightarrow Y$, для которых величина $\mathfrak{R}\left(\mathfrak{H}, \mathrm{F} ; \mathcal{I}^{a}, \mathcal{J}^{a}, \Upsilon^{\prime a} ; Y\right)$ близка к $\mathfrak{S}^{\prime}(\mathfrak{H}, \mathrm{F} ; \mathfrak{I}, \mathfrak{J} ; Y)$ в том или ином смысле.

Рассмотрим следующий простой

П р и м е р. Пусть $\mathrm{F}=W_{2}^{s}(\mathbb{T})(s \in \mathbb{N})$ - класс Соболева функций $f: \mathbb{T} \rightarrow \mathbb{C}$, имеющих абсолютно непрерывную $(s-1)$-производную и таких, что $\left\|\left.f\left|W_{2}^{s}(\mathbb{T}) \|^{2}:=\right| \widehat{f}(0)\right|^{2}+\right.$ $\left\|f^{(s)} \mid L_{2}(\mathbb{T})\right\|^{2} \leq 1 ; Y=L_{r}(\mathbb{T}):(1 \leq r \leq \infty)$; оператор информации $\mathcal{I}=\mathcal{I}_{\Xi} W_{2}^{s}(\mathbb{T}) \rightarrow \mathbb{C}^{\# \Xi}: f \mapsto$ $(\widehat{f}(\xi): \xi \in \Xi)$, где $\widehat{f}(\xi)$ - тригонометрические коэффициенты Фурье функции $f$ и $\varnothing \neq \Xi \subset \mathbb{Z}$ конечно. Тогда легко проверить, что для любого вектора $a_{\Xi}=\left(a_{\xi}: \xi \in \Xi\right) \in \mathcal{I}\left(W_{2}^{s}(\mathbb{T})\right)$ тригонометрический полином $t(x)=\sum_{\xi \in \Xi} a_{\xi} e^{2 \pi i \xi x}$ принадлежит $W_{2}^{s}(\mathbb{T})$ и является центром симметрии множества $\mathcal{I}^{-1}\left(a_{\Xi}\right)$. Поэтому и в силу $(1.2)$ для любого линейного (необязательно ограниченного) оператора $T: L_{p}(\mathbb{T}) \rightarrow L_{r}(\mathbb{T})(1 \leq p \leq \infty)$ (линейный) центральный алгоритм

$$
\Upsilon^{o}: W_{2}^{s}(\mathbb{T}) \rightarrow L_{r}(\mathbb{T}): f \mapsto \sum_{\xi \in \Xi} \widehat{f}(\xi) T\left(e^{2 \pi i \xi x}\right)
$$

является оптимальным методом восстановления оператора $T$ в задаче (1.1).

Если $T$ - оператор $T^{g}$ свертки с "хорошим" распределением $g \in \mathcal{S}^{\prime}(\mathbb{T})$, то оптимальным методом является

$$
\Upsilon^{o}(\mathcal{I}(f), x)=\sum_{\xi \in \Xi} \widehat{f}(\xi) \widehat{g}(\xi) e^{2 \pi i \xi x}
$$

и он "использует" столько же информации $\mathcal{J}(T)=(\widehat{g}(\xi): \xi \in \Xi)$ об операторе $T$, сколько и о функции $f$.

Если же $T$ - дифференциальный оператор $T_{h}=h(x) \frac{d^{k}}{d x^{k}}$ с достаточно гладким коэффициентом $h: \mathbb{T} \rightarrow \mathbb{C}$, который не является тригонометрическим полиномом (т.е. множество $\mathbb{Z}(h):=\{\xi \in \mathbb{Z} \mid \widehat{h}(\xi) \neq 0\}$ счетно), то оптимальный метод принимает вид

$$
\Upsilon^{o}(\mathcal{I}(f), x)=\sum_{\xi \in \Xi} \sum_{\zeta \in \mathbb{Z}} \widehat{f}(\xi) \widehat{h}(\zeta) e^{2 \pi i(\xi+\zeta) x}
$$

и "использует" уже бесконечную "информацию" об операторе $T$ (именно, весь бесконечный набор $(\widehat{h}(\xi): \xi \in \mathbb{Z}(h))$ коэффициентов Фурье функции $h)$.

Так как операторы являются объектами заведомо не менее "сложными", чем элементы (функции), на которые они действуют, а потому и информация о них в задачах, интересных для приложений, не обязана быть полной (во всяком случае, "намного более полной", чем информация о функциях), то ввиду примера оператора $T_{h}$ представляется вполне разумным рассматривать и исследовать постановки задач оптимального восстановления (классов) операторов, в которых информация об операторах также является неполной.

В следующих разделах изучается задача (1.5) для одного класса тороидальных псевдодифференциальных операторов и классов распределений типа Никольского - Бесова и Лизоркина - Трибеля и семейств операторов спектральной информации о символах ПДО и о распределениях. При этом используются обозначения, введенные в части I данной работы, в частности, $\mathbb{N}_{0}^{n}, \mathrm{z}_{n},|\mathrm{~m}|-\ell_{1}$-норма вектора т и др.

\section{2. Оптимальное восстановление классов ПДО}

Пусть заданы числа $m, n \in \mathbb{N}, n \leq m$, и вектор $\mathrm{m}=\left(m_{1}, \ldots, m_{n}\right) \in \mathbb{N}^{n}$ с $|\mathrm{m}|=m$. Далее, пусть $1 \leq p, q \leq \infty, 1<r<\infty, s=\left(s_{1}, \ldots, s_{n}\right) \in \mathbb{R}^{n}$. 
Рассмотрим в качестве $Y$ пространство $L_{r}=L_{r}\left(\mathbb{T}^{m}\right)$, в качестве $\mathrm{F}$ - классы распределений $\mathrm{B}_{p q}^{s \mathrm{~m}}\left(\mathbb{T}^{m}\right)$ типа Никольского - Бесова и $\mathrm{L}_{p q}^{s \mathrm{~m}}\left(\mathbb{T}^{m}\right)$ типа Лизоркина-Трибеля $(p<\infty)$ (см. определение 2 из части I $)^{2}$.

Основной объект исследования здесь (как и в первой части работы) - псевдодифференциальные операторы на $m$-мерном торе ((тороидальные) ПДО) вида

$$
T_{a}: f \mapsto T_{a} f(x)=\sum_{\xi \in \mathbb{Z}^{m}} a(x, \xi) \widehat{f}(\xi) e^{2 \pi i \xi x}
$$

с символами $a: \mathbb{T}^{m} \times \mathbb{Z}^{m} \rightarrow \mathbb{C}$ из классов $\widetilde{\Psi}_{\epsilon \vartheta}^{\tau \mathrm{m}}[v ; \mathrm{K}, \mathrm{L}] \equiv \Psi_{\epsilon \vartheta}^{\tau \mathrm{m}}[v ; \mathrm{K}, \mathrm{L}]\left(\mathbb{T}^{m} \times \mathbb{Z}^{m}\right)$. Здесь $1 \leq \theta \leq \infty$, $\tau=\left(\tau_{1}, \ldots, \tau_{n}\right), 0=(0, \ldots, 0), 1=(1, \ldots, 1) \in \mathbb{R}^{n}, v=\left(v_{1}, \ldots, v_{n}\right) \in \mathbb{R}_{+}^{n}, \epsilon=\left(\epsilon_{1}, \ldots, \epsilon_{n}\right) \in[0,1]^{n}$, $\mathrm{K}=\left(\mathrm{K}_{1}, \ldots, \mathrm{K}_{n}\right), \mathrm{L}=\left(\mathrm{L}_{1}, \ldots, \mathrm{L}_{n}\right) \in \mathbb{N}_{0}^{n}$.

Более точно, мы рассматриваем задачу (1.5) для класса операторов

$$
\mathfrak{H}_{\epsilon \vartheta}^{\tau \mathrm{m}}[v ; \mathrm{K}, \mathrm{L}]=\left\{T_{a}\left|a \in \widetilde{\Psi}_{\epsilon \vartheta}^{\tau \mathrm{m}}[v ; \mathrm{K}, \mathrm{L}]:\left\|a\left|\widetilde{S}_{1,0}^{\tau \mathrm{m}}[\mathrm{K}, \mathrm{L}]\|+\| a\right| \widetilde{\Psi}_{\epsilon \theta}^{\tau \mathrm{m}}[v ; \mathrm{K}, \mathrm{L}]\right\| \leq 1\right\}\right.
$$

(определения пространств тороидальных символов $\widetilde{S}_{1,0}^{\tau \mathrm{m}}[\mathrm{K}, \mathrm{L}]$ и $\widetilde{\Psi}_{\epsilon \theta}^{\tau \mathrm{m}}[v ; \mathrm{K}, \mathrm{L}]$ и их норм даны в определениях 1 и 3 части I).

Через $\mathfrak{D}$ обозначим набор функционалов $\left\{\mathfrak{d}_{\xi} \mid \xi \in \mathbb{Z}^{m}\right\}$, где $\mathfrak{d}_{\xi}(f)=\widehat{f}(\xi)-$ коэффициенты Фурье распределения $f \in \mathcal{S}^{\prime}\left(\mathbb{T}^{m}\right)$, через $\mathfrak{G}$ - набор функционалов $\left\{\mathfrak{g}_{\xi, \zeta} \mid(\xi, \zeta) \in \mathbb{Z}^{m} \times \mathbb{Z}^{m}\right\}$, где $\mathfrak{g}_{\xi, \zeta}(a)=\widehat{a}(\zeta, \xi)$ - коэффициенты Фурье символа $a(\cdot, \xi)$.

Тогда для фиксированного $N \in \mathbb{N}$ через $\mathfrak{I}_{N}$ обозначим семейство операторов информации $\left\{\mathcal{I}_{\Lambda} \mid \Lambda \subset \mathbb{Z}^{m}: \# \Lambda=N\right\}$, где $\mathcal{I}_{\Lambda}=\left(\mathfrak{d}_{\xi}: \xi \in \Lambda\right)$, а через $\mathfrak{J}-$ семейство операторов информации $\left\{\mathcal{J}_{\mathrm{M}} \mid \mathrm{M} \subset \mathbb{Z}^{m} \times \mathbb{Z}^{m}: \# \mathrm{M}<\infty\right\}$, где $\mathcal{J}_{\mathrm{M}}=\left(\mathfrak{g}_{\xi, \zeta}:(\xi, \zeta) \in \mathrm{M}\right)$.

Наконец, по параметрам $p, r$ и векторам $s, \tau$ определим числа

$$
\sigma_{\nu}=\frac{s_{\nu}-\tau_{\nu}}{m_{\nu}}, \quad \nu \in \mathrm{z}_{n}, \quad \sigma:=\min \left\{\sigma_{\nu}: \nu \in \mathrm{z}_{n}\right\}, \quad \omega=\#\left\{\nu \in \mathrm{z}_{n}: \sigma_{\nu}=\sigma\right\}
$$

$\varsigma=\sigma-\left(\frac{1}{p}-\frac{1}{r}\right)_{+}$. Здесь $u_{+}=\max \{u, 0\}$ для $u \in \mathbb{R}$.

Будем использовать знаки «и $\asymp$ порядкового неравенства и равенства: для функций $F: \mathbb{R}_{+} \rightarrow \mathbb{R}_{+}$и $H: \mathbb{R}_{+} \rightarrow \mathbb{R}_{+}$пишем $F(u) \ll H(u)$ при $u \rightarrow \infty$, если найдется такая константа $C=C(F, H)>0$, что верно неравенство $F(u) \leq C H(u)$ для $u \geq u_{0}>0 ; F(u) \asymp H(u)$, если одновременно $F(u) \ll H(u)$ и $H(u) \ll F(u)$. Часто вместо слов "порядковое равенство" будем говорить "порядковая оценка", подразумевая порядковую двустороннюю оценку.

Следующая теорема - главный результат работы (наряду с теоремой 3, см. разд. 4 ниже), в ней при естественных соотношениях между параметрами задачи дается точный порядок $($ по $N)$ величины $(1.5)$ в нашей задаче оптимального восстанвления класса ПДО $\mathfrak{H}_{\epsilon \vartheta}^{\tau \mathrm{m}}[v ; \mathrm{K}, \mathrm{L}]$ по семействам операторов информации $\mathfrak{I}_{N}$ и $\mathfrak{J}$ на классах типа Никольского - Бесова и Лизоркина - Трибеля в пространстве $L_{r}$.

Теорема 1. Предположим, что $1 \leq p, q, \theta \leq \infty, 1<r<\infty, s, \tau \in \mathbb{R}^{n}, v \in \mathbb{R}_{+}^{n}, \epsilon \in[0,1]^{n}$ такие, что $\varsigma>0$, и для любого $\nu \in \mathrm{z}_{n}$ выполнено одно из следующих трех условий:

(i) $s_{\nu}-\tau_{\nu}<v_{\nu}$;

(ii) $s_{\nu}-\tau_{\nu}=v_{\nu}, \quad \epsilon_{\nu}<1 \quad u \quad \theta \leq q$;

(iii) $s_{\nu}-\tau_{\nu}=v_{\nu}, \quad \epsilon_{\nu}=\theta=q=1$.

Положим

$$
\mathrm{K}_{f}=\left(f\left\lfloor\frac{m_{1}}{2}\right\rfloor+f+4, \ldots, f\left\lfloor\frac{m_{n}}{2}\right\rfloor+f+4\right), \quad f \in\{b=3, I=5\}, \quad \mathrm{L}=\left(\left\lfloor\frac{m_{1}}{r}\right\rfloor, \ldots,\left\lfloor\frac{m_{n}}{r}\right\rfloor\right) .
$$

\footnotetext{
${ }^{2}$ Всюду ниже при рассмотрении классов типа Лизоркина - Трибеля будем считать, не оговаривая этого специально, что $p<\infty$.
} 
Тогда для $(\mathrm{F}, f) \in\{(\mathrm{B}, b),(\mathrm{L}, /)\}$ верна следующая оченка сверху:

$$
\mathfrak{S}\left(\mathfrak{H}_{\varepsilon \vartheta}^{\tau \mathrm{m}}\left[v ; \mathrm{K}_{f}, \mathrm{~L}\right], \mathrm{F}_{p q}^{s \mathrm{~m}} ; \mathfrak{I}_{N}, \mathfrak{J} ; L_{r}\right) \ll \varphi_{N}\left(\mathrm{~F}_{p q}^{s-\tau \mathrm{m}}, L_{r}\right), \quad N \rightarrow \infty .
$$

Далее,

а) если, кроме того, $2 \leq p, q \leq \infty, r \leq p$, то верна соответствующая оценка снизу

$$
\mathfrak{S}\left(\mathfrak{H}_{\varepsilon \vartheta}^{\tau \mathrm{m}}\left[v ; \mathrm{K}_{f}, \mathrm{~L}\right], \mathrm{F}_{p q}^{s \mathrm{~m}} ; \mathfrak{I}_{N}, \mathfrak{J} ; L_{r}\right) \gg \varphi_{N}\left(\mathrm{~F}_{p q}^{s-\tau \mathrm{m}}, L_{r}\right), \quad N \rightarrow \infty ;
$$

b) если, кроме того, $1 \leq q<2 \leq p \leq \infty, r \leq p$, то верна следующая оченка снизу:

$$
\mathfrak{S}\left(\mathfrak{H}_{\varepsilon \vartheta}^{\tau \mathrm{m}}\left[v ; \mathrm{K}_{f}, \mathrm{~L}\right], \mathrm{F}_{p q}^{s \mathrm{~m}} ; \mathfrak{I}_{N}, \mathfrak{J} ; L_{r}\right) \gg \varphi_{N}\left(\mathrm{~F}_{p q}^{s-\tau \mathrm{m}}, L_{r}\right)\left(\log ^{\omega-1} N\right)^{(1 / 2-1 / q)}, \quad N \rightarrow \infty,
$$

а также точная порядковая оценка для линейного оптимального восстановления

$$
\mathfrak{S}^{\prime}\left(\mathfrak{H}_{\varepsilon \vartheta}^{\tau \mathrm{m}}\left[v ; \mathrm{K}_{f}, \mathrm{~L}\right], \mathrm{F}_{p q}^{s \mathrm{~m}} ; \mathfrak{I}_{N}, \mathfrak{J} ; L_{r}\right) \asymp \varphi_{N}\left(\mathrm{~F}_{p q}^{s-\tau \mathrm{m}}, L_{r}\right), \quad N \rightarrow \infty ;
$$

с) если, кроме того, $1<p<r \leq 2,1 \leq q \leq \infty$, то верна следующая оченка снизу:

$$
\mathfrak{S}\left(\mathfrak{H}_{\varepsilon \vartheta}^{\tau \mathrm{m}}\left[v ; \mathrm{K}_{f}, \mathrm{~L}\right], \mathrm{F}_{p q}^{s \mathrm{~m}} ; \mathfrak{I}_{N}, \mathfrak{J} ; L_{r}\right) \gg \varphi_{N}\left(\mathrm{~F}_{p q}^{s-\tau \mathrm{m}}, L_{r}\right)\left(\log ^{\omega-1} N\right)^{(1 / 2-1 / q)}, \quad N \rightarrow \infty,
$$

а также точная порядковая оценка для линейного оптимального восстановления

$$
\mathfrak{S}^{\prime}\left(\mathfrak{H}_{\varepsilon \vartheta}^{\tau \mathrm{m}}\left[v ; \mathrm{K}_{f}, \mathrm{~L}\right], \mathrm{F}_{p q}^{s \mathrm{~m}} ; \mathfrak{I}_{N}, \mathfrak{J} ; L_{r}\right) \asymp \varphi_{N}\left(\mathrm{~F}_{p q}^{s-\tau \mathrm{m}}, L_{r}\right), \quad N \rightarrow \infty
$$

d) если, кроме того, $2 \leq p<r<\infty, 1 \leq q \leq \infty$, то верна следующая оченка снизу:

$$
\mathfrak{S}\left(\mathfrak{H}_{\varepsilon \vartheta}^{\tau \mathrm{m}}\left[v ; \mathrm{K}_{f}, \mathrm{~L}\right], \mathrm{F}_{p q}^{s \mathrm{~m}} ; \mathfrak{I}_{N}, \mathfrak{J} ; L_{r}\right) \gg \varphi_{N}\left(\mathrm{~F}_{p q}^{s-\tau \mathrm{m}}, L_{r}\right)\left(\log ^{\omega-1} N\right)^{(1 / 2-1 / q)}, \quad N \rightarrow \infty,
$$

а также точная порядковая оценка для линейного оптимального восстановления

$$
\mathfrak{S}^{\prime}\left(\mathfrak{H}_{\varepsilon \vartheta}^{\tau \mathrm{m}}\left[v ; \mathrm{K}_{f}, \mathrm{~L}\right], \mathrm{F}_{p q}^{s \mathrm{~m}} ; \mathfrak{I}_{N}, \mathfrak{J} ; L_{r}\right) \asymp \varphi_{N}\left(\mathrm{~F}_{p q}^{s-\tau \mathrm{m}}, L_{r}\right), \quad N \rightarrow \infty
$$

е) если, кроме того, $1 \leq r \leq p<2,1 \leq q \leq \infty$, то верна следующая оченка снизу:

$$
\mathfrak{S}\left(\mathfrak{H}_{\varepsilon \vartheta}^{\tau \mathrm{m}}\left[v ; \mathrm{K}_{f}, \mathrm{~L}\right], \mathrm{F}_{p q}^{s \mathrm{~m}} ; \mathfrak{I}_{N}, \mathfrak{J} ; L_{r}\right) \gg\left(\frac{\log ^{\omega-1} N}{N}\right)^{\sigma}\left(\log ^{\omega-1} N\right)^{(1 / 2-1 / q)}, \quad N \rightarrow \infty .
$$

Здесъ $\varphi_{N}\left(\mathrm{~F}, L_{r}\right)-N$-й поперечник Фуръе множества $\mathrm{F}$ в пространстве $L_{r}$.

3 а м е ч а н и е 1 . Напомним, что $N$-й поперечник Фурье множества $\mathrm{F}\left(\subset L_{r}\right)$ в пространстве $L_{r}$ определяется по формуле

$$
\varphi_{N}\left(\mathrm{~F}, L_{r}\right)=\inf \left\{\sup \left\{\left\|f-\sum_{k=1}^{N}\left\langle f, g_{k}\right\rangle g_{k}\left|L_{r} \|\right| f \in \mathrm{F}\right\} \mid\left(g_{k}\right)_{k=1}^{N}\right\},\right.
$$

где нижняя грань берется по всевозможным ортонормированным системам из $N$ функций $\left(g_{k}\right)_{k=1}^{N} \subset L_{\infty},\langle f, h\rangle=\int_{\mathbb{T}^{m}} f(x) \bar{h}(x) d x$.

Поперечники Фурье были введены В. Н. Темляковым в 1982 г. Весьма обстоятельный обзор результатов по поперечникам Фурье различных функциональных классов можно найти в недавней монографии [7, Ch. 4]. Точные по порядку оценки поперечников Фурье функциональных классов типа Никольского - Бесова и Лизоркина - Трибеля, рассматриваемых здесь, во всей их полноте были установлены автором в 2010 г. (см. [8], где доказаны оценки сверху, и работу [9], где получены соответствующие оценки снизу); в [9] также имеются достаточно 
подробная история вопроса и библиография. В частности, в условиях теоремы 1 справедливы оценки $(\mathrm{c}(\mathrm{F}, \mathrm{f}(p, q, r)) \in\{(\mathrm{B}, \mathrm{b}(p, q, r)),(\mathrm{L}, \mathrm{I}(p, q, r))\})$

$$
\varphi_{N}\left(\mathrm{~F}_{p q}^{s-\tau \mathrm{m}}, L_{r}\right) \asymp\left(\frac{\log ^{\omega-1} N}{N}\right)^{\varsigma}\left(\log ^{\omega-1} N\right)^{\mathrm{f}(p, q, r)},
$$

где

при $r \leq p$ и

$$
\mathrm{b}(p, q, r)=\left(\frac{1}{p_{*}}-\frac{1}{q}\right)_{+}, \quad \mathrm{I}(p, q, r)=\left(\frac{1}{2}-\frac{1}{q}\right)_{+}
$$

$$
\mathrm{b}(p, q, r)=\left(\frac{1}{r}-\frac{1}{q}\right)_{+}, \quad \mathrm{I}(p, q, r)=0
$$

при $p<r$; здесь $p_{*}=\min \{p, 2\}$.

\section{3. Тригонометрические полиномы. Оператор лифтинга}

Пусть $\Lambda-$ произвольное конечное множество из $\mathbb{Z}^{m}$ и

$$
\mathrm{T}(\Lambda)=\left\{t(x)=\sum_{\xi \in \Lambda} \widehat{t}(\xi) e^{2 \pi i \xi x} \mid \widehat{t}(\xi) \in \mathbb{C}, \xi \in \Lambda\right\}
$$

- пространство тригонометрических полиномов со спектром $\Lambda$.

Для распределения $f \in \mathcal{S}^{\prime}\left(\mathbb{T}^{m}\right)$ пусть, как и раньше,

$$
f(x)=\sum_{\xi \in \mathbb{Z}^{m}} \widehat{f}(\xi) e^{2 \pi i \xi x}, \quad S_{\Lambda}(f, x)=\sum_{\xi \in \Lambda} \widehat{f}(\xi) e^{2 \pi i \xi x} \quad(\in \mathrm{T}(\Lambda))
$$

- ее ряд Фурье и сумма Фурье, соответствующая спектру $\Lambda$.

Положим

$$
\rho_{+}(\mathrm{m}, \kappa)=\left\{\xi \in \mathbb{N}^{m}: 2^{\kappa_{\nu}-1} \leq \xi_{k} \leq 2^{\kappa_{\nu}}, k=k_{\nu-1}+1, \ldots, k_{\nu}, \nu \in \mathrm{z}_{n}\right\}
$$

(напомним, что здесь $k_{0}=0, k_{\nu}=m_{1}+\cdots+m_{\nu}, \nu \in \mathrm{z}_{n}$ ) и для четного $u \in \mathbb{N}$

$$
\vartheta[u] \equiv \vartheta[u ; a] \equiv\left\{\kappa \in \mathbb{N}^{n} \mid u \leq \kappa \mathrm{m} \leq u+2 a, \kappa_{\nu} \text { - четные, } \nu \in \mathrm{z}_{n}\right\}, \quad \Lambda_{+}(u) \equiv \cup_{\kappa \in \vartheta[u]} \rho_{+}(\mathrm{m}, \kappa) .
$$

В [9] получены оценки

$$
\# \vartheta[u] \asymp u^{n-1}, \quad \# \Lambda_{+}(u) \asymp 2^{u} u^{n-1} .
$$

Следующая теорема, которую мы применим при получении оценок снизу в теореме 1 , является частным случаем теоремы Т1, доказанной в [9] и обобщающей известный результат В. Н. Темлякова [10].

Теорема 2. Для любого множества $\Lambda \subset \mathbb{Z}^{m}$ такого, что $\# \Lambda \leq \frac{1}{2} \# \Lambda_{+}(u)$, существует полином $t_{\Lambda, u} \in \mathrm{T}\left(\Lambda_{+}(u) \backslash \Lambda\right)$ такой, что для всех $\kappa \in \vartheta[u]$

$$
\left\|\sum_{\xi \in \rho_{+}(\mathrm{m}, \kappa)} \widehat{t}_{\Lambda, u}(\xi) e^{2 \pi i \xi x}\left|L_{\infty}\left\|\leq(\# \vartheta[u])^{-1 / 2} u\right\| t_{\Lambda, u}\right| L_{2}\right\| \geq c(\mathrm{~m})>0 .
$$

В дальнейшем нам также понадобятся оценки нормы полинома из теоремы 2 в пространствах типа Никольского - Бесова и Лизоркина - Трибеля :

$$
\left\|t_{\Lambda, u} \mid F_{p q}^{s-\tau \mathrm{m}}\right\| \ll 2^{\sigma u} u^{(\omega-1)(1 / q-1 / 2)} .
$$

Оценка (3.3) для пространств типа Никольского - Бесова (случай $F=B$ ) получена в [9]; для пространств типа Лизоркина - Трибеля (случай $F=L$ ) оценка устанавливается аналогично. 
Теперь рассмотрим символ (не зависящий от пространственной переменной $x$ )

$$
a^{(\tau)}(\xi):=\prod_{\nu \in \mathrm{Z}_{n}}\left\langle\xi^{\nu}\right\rangle^{\tau_{\nu}} \quad\left(\xi \in \mathbb{Z}^{m}\right),
$$

который принадлежит классу $\Psi_{\epsilon \vartheta}^{\tau \mathrm{m}}\left[v ; \mathrm{K}_{\mathrm{f}}, \mathrm{L}\right]:$ во-первых, $a^{(\tau)}(\xi) \in \widetilde{S}_{1,0}^{\tau \mathrm{m}}[\mathrm{K}, \mathrm{L}]$ (см., например, [11, Remark 2.1.4, p. 261]); во-вторых, нетрудно показать, что любой символ $b(\xi) \in \widetilde{S}_{1,0}^{\tau \mathrm{m}}[\mathrm{K}, \mathrm{L}]$, который не зависит от $x$, принадлежит классу $\Psi_{\epsilon \vartheta}^{\tau \mathrm{m}}[v ; \mathrm{K}, \mathrm{L}]$. Поэтому оператор $T_{a(\tau)}$ принадлежит классу $\mathfrak{H}_{\varepsilon \vartheta}^{\tau \mathrm{m}}\left[v ; \mathrm{K}_{\mathrm{f}}, \mathrm{L}\right]$.

С другой стороны, хорошо известно, что оператор $I^{(\tau)}\left(\equiv T_{a^{(\tau)}}\right)$ (называемый оператором лифтинга) играет важную роль в теории функциональных пространств, в частности, осуществляет изоморфизм между пространствами $F_{p q}^{s \mathrm{~m}}$ and $F_{p q}^{s-\tau \mathrm{m}}$, другими словами, отображение

$$
I^{(\tau)}: F_{p q}^{s \mathrm{~m}} \rightarrow F_{p q}^{s-\tau \mathrm{m}}: f \mapsto g:=I^{(\tau)}(f)
$$

биективно и, кроме того, $\left\|f\left|F_{p q}^{s \mathrm{~m}}\|\asymp\| g\right| F_{p q}^{s-\tau \mathrm{m}}\right\|$ (см., например, [12, гл. 8, п. 8.8; 13, гл. 2, п. 2.3.8; 14, Ch.2, Sect.2.2.6]).

Ясно, что

$$
\widehat{g}(\xi)=\prod\left\langle\xi^{\nu}\right\rangle^{\tau_{\nu}} \widehat{f}(\xi), \quad \xi \in \mathbb{Z}^{m},
$$

и поэтому $\widehat{g}(\xi)=0 \Leftrightarrow \widehat{f}(\xi)=0$. Следовательно,

$\sup \left\{\left\|I^{(\tau)} f\left|L_{r} \|\right| f \in \mathrm{F}_{p q}^{s \mathrm{~m}}: \widehat{f}(\xi)=0, \xi \in \Xi\right\} \asymp \sup \left\{\left\|g\left|L_{r} \|\right| g \in \mathrm{F}_{p q}^{s-\tau \mathrm{m}}: \widehat{g}(\xi)=0, \xi \in \Xi\right\}\right.\right.$.

\section{4. Доказательство теоремы 1: оценки сверху}

Напомним конструкцию линейного метода восстановления $\Upsilon_{\Lambda(\gamma, N)}$ из части I.

Прежде всего определим вектор $\gamma=\left(\gamma_{1}, \ldots, \gamma_{n}\right) \in \mathbb{R}^{n}$. Не ограничивая общности, считаем, что $\varsigma=\varsigma_{1}=\ldots=\varsigma_{\omega}<\varsigma_{\nu}, \nu \in \mathrm{z}_{n} \backslash \mathrm{z}_{\omega}$. Выберем числа $\varsigma_{\nu}^{\prime}, \nu \in \mathrm{z}_{n}$, из условий $\varsigma=\varsigma_{1}^{\prime}=\ldots=\varsigma_{\omega}^{\prime}$, $\varsigma<\varsigma_{\nu}^{\prime}<\varsigma_{\nu}$ при $\nu \in \mathrm{z}_{n} \backslash \mathrm{z}_{\omega}$. Наконец, положим $\gamma_{\nu}=\varsigma_{\nu}^{\prime} m_{\nu} / \varsigma, \nu \in \mathrm{z}_{n}$.

По $\gamma$ и $u \in \mathbb{R}_{+}$определим спектры $\Lambda_{u}^{\gamma} \subset \mathbb{Z}^{m}, \Lambda_{u}^{\gamma, \eta} \subset \mathbb{Z}^{m}$, полагая

$$
\Lambda_{u}^{\gamma}:=\cup_{\kappa \in \mathbb{N}_{0}^{n}: \gamma \kappa \leq u} \rho(\mathrm{m}, \kappa), \quad \Lambda_{u}^{\gamma, \eta}:=\cup_{\kappa \in \mathbb{N}_{0}^{n}: \gamma \kappa \leq u} \rho(\mathrm{m}, \eta, \kappa),
$$

где

$$
\begin{gathered}
\rho(\mathrm{m}, \kappa):=\left\{\left.\xi \in \mathbb{Z}^{m}\left|\left\lfloor 2^{\kappa_{\nu}-1}\right\rfloor \leq\right| \xi^{\nu}\right|_{\infty}<2^{\kappa_{\nu}}, \nu \in \mathrm{z}_{n}\right\}, \\
\rho(\mathrm{m}, \eta, \kappa):=\left\{\left.\xi \in \mathbb{Z}^{m}\left|\left\lfloor 2^{\kappa_{\nu}-1}\right\rfloor \leq\right| \xi^{\nu}\right|_{\infty}<3 \cdot 2^{\kappa_{\nu}-1}, \nu \in \mathrm{z}_{n}\right\} \quad\left(\kappa \in \mathbb{N}_{0}^{n}\right)
\end{gathered}
$$

$(\lfloor u\rfloor$ - целая часть числа $u \in \mathbb{R})$. В части I установлены включения $\left(u(\gamma):=\gamma_{1}+\ldots+\gamma_{n}\right)$

$$
\Lambda_{u}^{\gamma} \subset \Lambda_{u}^{\gamma, \eta} \subset \Lambda_{u+u(\gamma)}^{\gamma}
$$

и оценка $\# \Lambda_{u}^{\gamma} \asymp 2^{u} u^{\omega-1}$. Для любого $N \in \mathbb{N}$ существует единственное $u_{N} \in \mathbb{R}_{+}$такое, что $N=2^{u_{N}} u_{N}^{\omega-1}$. Обозначим $\Lambda(\gamma, N):=\Lambda_{u_{N}}^{\gamma}$, т.е. $\# \Lambda(\gamma, N) \asymp N$.

Тогда метод $\Upsilon_{\Lambda(\gamma, N)}$ восстановления ПДО $T_{a}$, использующий операторы информации $\mathcal{I}_{\Lambda(\gamma, N)}$ и $\mathcal{J}_{\mathrm{M}(\gamma, N)}, \mathrm{M}(\gamma, N)=\{(\xi, \zeta) \mid \xi \in \Lambda(\gamma, N), \zeta \in \Lambda(\gamma, N)-\xi\}$ (в обозначениях части I $\mathfrak{D}_{\Lambda(\gamma, N)}$ и $\mathfrak{G}_{\Lambda(\gamma, N)}$ соответственно), определяется по формуле

$$
\Upsilon_{\Lambda(\gamma, N)}\left(\mathcal{I}_{\Lambda(\gamma, N)}(f), \mathcal{J}_{\mathrm{M}(\gamma, N)}\left(T_{a}\right)\right)(x):=S_{\Lambda(\gamma, N)}\left(T_{a} S_{\Lambda(\gamma, N)}(f), x\right) .
$$

Метод восстановления $\Upsilon_{\Lambda(\gamma, N)}$ использует $M:=\# \Lambda(\gamma, N)+(\# \Lambda(\gamma, N))^{2} \asymp N^{2}$ "единиц информации": $(\# \Lambda(\gamma, N))^{2}$ - об операторе, $\# \Lambda(\gamma, N)$ - о функции.

Оценка сверху (2.2), а также оценки сверху в соотношениях $(2.5),(2.7),(2.9)$ в теореме 1 будут следовать из следующей теоремы, в которой устанавливается точный порядок (по $N$ ) величины погрешности (1.4) (линейного) метода $\Upsilon_{\Lambda(\gamma, N)}$ восстановления ПДО $T_{a}$ вида (2.1) по оператору $\mathcal{I}_{\Lambda(\gamma, N)}$ информации об $f \in \mathrm{F}_{p q}^{s \mathrm{~m}}(\mathrm{~F} \in\{\mathrm{B}, \mathrm{L}\})$ и оператору $\mathcal{J}_{\mathrm{M}(\gamma, N)}$ информации о символах $a \in \widetilde{\Psi}_{\epsilon \theta}^{\tau \mathrm{m}}[v ; \mathrm{K}, \mathrm{L}]$ в пространстве $L_{r}$. 
Теорема 3. Предположим, что $1 \leq p, q, \theta \leq \infty, 1<r<\infty, s, \tau \in \mathbb{R}^{n}, v \in \mathbb{R}_{+}^{n}, \epsilon \in[0,1]^{n}$ такие, что $\varsigma>0$ и для любого $\nu \in \mathrm{z}_{n}$ выполнено одно из следующих трех условий:

(i) $s_{\nu}-\tau_{\nu}<v_{\nu}$

(ii) $s_{\nu}-\tau_{\nu}=v_{\nu}, \quad \epsilon_{\nu}<1 \quad u \quad \theta \leq q$;

(iii) $s_{\nu}-\tau_{\nu}=v_{\nu}, \quad \epsilon_{\nu}=\theta=q=1$.

Далее, пусть

$$
\mathrm{K}_{f}=\left(f\left\lfloor\frac{m_{1}}{2}\right\rfloor+f+4, \ldots, f\left\lfloor\frac{m_{n}}{2}\right\rfloor+f+4\right), f \in\{b=3, l=5\}, \quad \mathrm{L}=\left(\left\lfloor\frac{m_{1}}{r}\right\rfloor, \ldots,\left\lfloor\frac{m_{n}}{r}\right\rfloor\right) .
$$

Тогда для $(\mathrm{F}, f) \in\{(\mathrm{B}, b),(\mathrm{L}, /)\}$ верна оченка

$$
\mathfrak{R}\left(\mathfrak{H}_{\varepsilon \vartheta}^{\tau \mathrm{m}}\left[v ; \mathrm{K}_{f}, \mathrm{~L}\right], \mathrm{F}_{p q}^{s \mathrm{~m}} ; \mathcal{I}_{\Lambda(\gamma, N)}, \mathcal{J}_{\mathrm{M}(\gamma, N)}, \Upsilon_{\Lambda(\gamma, N)} ; L_{r}\right) \asymp \varphi_{N}\left(\mathrm{~F}_{p q}^{s-\tau \mathrm{m}}, L_{r}\right), \quad N \rightarrow \infty .
$$

3 а м е ч а н и е 2. Теорема 3 усиливает результат теоремы 1 из части I: во-первых, по сравнению с теоремой 1 в теореме 3 оценка сверху доказывается при "естественных", условиях на "дифференциальные", параметры $s$ и $\tau$, а именно, для $s, \tau \in \mathbb{R}^{n}$ таких, что $s>\tau$; во-вторых, в теореме 3 устанавливается соответствующая оценка снизу.

Д о к а з а т е л ь с т в о теоремы 3. Сначала докажем оценку сверху в (4.3). Для любого символа $a \in \widetilde{\Psi}_{\epsilon \theta}^{\tau \mathrm{m}}[v ; \mathrm{K}, \mathrm{L}]$ определим символ $b$ равенством

$$
b(x, \xi)=a(x, \xi) a^{(-\tau)}(\xi)=a(x, \xi) \prod_{\nu \in z_{n}}\left\langle\xi^{\nu}\right\rangle^{-\tau_{\nu}},
$$

тогда нетрудно видеть, что $b \in \widetilde{\Psi}_{\epsilon \theta}^{0 \mathrm{~m}}[v ; \mathrm{K}, \mathrm{L}]$. Для распределения $f \in \mathcal{S}^{\prime}\left(\mathbb{T}^{m}\right)$, как и выше, положим $g=I^{(\tau)} f$. Тогда, очевидно, имеем $T_{a} f=T_{b} g$ и, следовательно, $T_{a} S_{\Lambda(\gamma, N)}(f)=T_{b} S_{\Lambda(\gamma, N)}(g)$. Поэтому

$$
S_{\Lambda(\gamma, N)}\left(T_{a} S_{\Lambda(\gamma, N)}(f)\right)=S_{\Lambda(\gamma, N)}\left(T_{b} S_{\Lambda(\gamma, N)}(g)\right)
$$

Отсюда находим

$$
\begin{aligned}
& \left\|T_{a} f-S_{\Lambda(\gamma, N)}\left(T_{a} S_{\Lambda(\gamma, N)}(f)\right)\left|L_{r}\|=\| T_{b} g-S_{\Lambda(\gamma, N)}\left(T_{b} S_{\Lambda(\gamma, N)}(g)\right)\right| L_{r}\right\| \\
\leq & \left\|T_{b} g-S_{\Lambda(\gamma, N)}\left(T_{b} g\right)\left|L_{r}\|+\| S_{\Lambda(\gamma, N)}\left(T_{b}\left(g-S_{\Lambda(\gamma, N)}(g)\right)\right)\right| L_{r}\right\|=: \Im_{1}+\Im_{2} .
\end{aligned}
$$

Сперва оценим $\Im_{1}$. Так как по теореме 2 из части I оператор $T_{b}: F_{p q}^{s-\tau \mathrm{m}} \rightarrow F_{p q}^{s-\tau \mathrm{m}}$ ограничен, то с учетом свойств оператора лифтинга из разд. 3 получим

$$
\begin{gathered}
\left\|T_{b} g\left|F_{p q}^{s-\tau \mathrm{m}}\|\leq\| T_{b}\right| F_{p q}^{s-\tau \mathrm{m}} \rightarrow F_{p q}^{s-\tau \mathrm{m}}\right\|\left\|g \mid F_{p q}^{s-\tau \mathrm{m}}\right\| \\
\asymp\left\|T_{b}\left|F_{p q}^{s-\tau \mathrm{m}} \rightarrow F_{p q}^{s-\tau \mathrm{m}}\|\| f\right| F_{p q}^{s \mathrm{~m}}\right\| \leq\left\|T_{b} \mid F_{p q}^{s-\tau \mathrm{m}} \rightarrow F_{p q}^{s-\tau \mathrm{m}}\right\|,
\end{gathered}
$$

поэтому $h:=T_{b} g$ принадлежит $\left\|T_{b} \mid F_{p q}^{s-\tau \mathrm{m}} \rightarrow F_{p q}^{s-\tau \mathrm{m}}\right\| \mathrm{F}_{p q}^{s-\tau \mathrm{m}}-$ шару пространства $F_{p q}^{s-\tau \mathrm{m}}$ радиуса $\left\|T_{b} \mid F_{p q}^{s-\tau \mathrm{m}} \rightarrow F_{p q}^{s-\tau \mathrm{m}}\right\|$. Следовательно, используя (5.2) из части I, применяя теорему 4.1 (см. еще замечание 4.1) работы [8] и учитывая определение $u_{N}$, имеем (следует еще принять во внимание (4.1))

$$
\begin{gathered}
\Im_{1} \ll\left\|T_{b} \mid F_{p q}^{s-\tau \mathrm{m}} \rightarrow F_{p q}^{s-\tau \mathrm{m}}\right\| \sup \left\{\left\|h-S_{\Lambda(\gamma, N)}(h)\left|L_{r} \|\right| h \in \mathrm{F}_{p q}^{s-\tau \mathrm{m}}\right\}\right. \\
\leq\left(1+C_{r, \mathrm{~m}} / c_{r, \mathrm{~m}}\right)\left\|T_{b} \mid F_{p q}^{s-\tau \mathrm{m}} \rightarrow F_{p q}^{s-\tau \mathrm{m}}\right\| \sup \left\{\left\|h-\sum_{\kappa \gamma \leq u_{N}-u(\gamma)} \Delta_{\kappa}^{\eta}(h)\left|L_{r} \|\right| h \in \mathrm{F}_{p q}^{s-\tau \mathrm{m}}\right\}\right. \\
\asymp\left(1+C_{r, \mathrm{~m}} / c_{r, \mathrm{~m}}\right)\left\|T_{b} \mid F_{p q}^{s-\tau \mathrm{m}} \rightarrow F_{p q}^{s-\tau \mathrm{m}}\right\|\left(\frac{\log ^{\omega-1} N}{N}\right)^{\varsigma}\left(\log ^{\omega-1} N\right)^{\mathrm{f}(p, q, r)} .
\end{gathered}
$$

Ннапомним, что операторы $\Delta_{\kappa}^{\eta}$ "гладких срезок" ряда Фурье - из определения функциональных пространств типа Никольского-Бесова и Лизоркина-Трибеля (см. определение 2 из части I). 
Оценим теперь $\Im_{2}$. В силу п. 2) замечания 4 из части I в условиях теоремы 3 оператор $T_{b}: L_{r} \rightarrow L_{r}$ ограничен. Отсюда ввиду (5.1) и (5.2) из части I (принимая во внимание (4.1)), снова применяя теорему 4.1 из [8] и учитывая определение $u_{N}$, получим

$$
\begin{gathered}
\Im_{2} \leq C_{r, \mathrm{~m}} / c_{r, \mathrm{~m}}\left\|T_{b}\left|L_{r} \rightarrow L_{r}\|\| g-S_{\Lambda(\gamma, N)}(g)\right| L_{r}\right\| \\
\leq\left(1+C_{r, \mathrm{~m}} / c_{r, \mathrm{~m}}\right)^{2}\left\|T_{b} \mid L_{r} \rightarrow L_{r}\right\| \sup \left\{\left\|h-\sum_{\kappa \gamma \leq u_{N}-u(\gamma)} \Delta_{\kappa}^{\eta}(h)\left|L_{r} \|\right| h \in \mathrm{F}_{p q}^{s-\tau \mathrm{m}}\right\} .\right. \\
\asymp\left(1+C_{r, \mathrm{~m}} / c_{r, \mathrm{~m}}\right)^{2}\left\|T_{b} \mid L_{r} \rightarrow L_{r}\right\|\left(\frac{\log ^{\omega-1} N}{N}\right)^{\varsigma}\left(\log ^{\omega-1} N\right)^{\mathrm{f}(p, q, r)} .
\end{gathered}
$$

Таким образом, требуемая оценка сверху в (4.3) вытекает из полученных соотношений (4.4) и (4.5).

Переходим теперь к доказательству соответствующих оценок снизу в (4.3). Для того чтобы установить оценку снизу в (4.3), достаточно указать "плохой" оператор $T_{a} \in \mathfrak{H}_{\varepsilon \vartheta}^{\tau \mathrm{m}}\left[v ; \mathrm{K}_{\mathrm{f}}, \mathrm{L}\right]$ такой, что погрешность метода восстановления $\Upsilon_{\Lambda(\gamma, N)}$ этого оператора на классе $\mathrm{F}_{p q}^{s m}$ будет иметь порядок соответствующего поперечника Фурье класса $\mathrm{F}_{p q}^{s-\tau \mathrm{m}}$. Оказывается, в качестве такого "плохого" оператора можно взять оператор лифтинга $I^{(\tau)}$.

Действительно, прежде всего легко видеть, что для любого распределения $f \in \mathcal{S}^{\prime}\left(\mathbb{T}^{m}\right)$

$$
S_{\Lambda(\gamma, N)}\left(I^{(\tau)} S_{\Lambda(\gamma, N)}(f)\right)=I^{(\tau)} S_{\Lambda(\gamma, N)}(f)=S_{\Lambda(\gamma, N)}\left(I^{(\tau)} f\right) .
$$

Поэтому (при $\left.g=I^{(\tau)} f\right)$ с учетом свойств оператора лифтинга и определения поперечника Фурье имеем

$$
\begin{gathered}
\sup \left\{\| g - S _ { \Lambda ( \gamma , N ) } ( g ) | L _ { r } \| | f \in \mathrm { F } _ { p q } ^ { s \mathrm { m } } \} \asymp \operatorname { s u p } \left\{\left\|g-S_{\Lambda(\gamma, N)}(g)\left|L_{r} \|\right| g \in \mathrm{F}_{p q}^{s-\tau \mathrm{m}}\right\}\right.\right. \\
\geq \varphi_{\# \Lambda(\gamma, N)}\left(\mathrm{F}_{p q}^{s-\tau \mathrm{m}}, L_{r}\right) \asymp \varphi_{N}\left(\mathrm{~F}_{p q}^{s-\tau \mathrm{m}}, L_{r}\right) .
\end{gathered}
$$

Итак, требуемая оценка снизу в соотношении (4.3), а вместе с ней и теорема 3 полностью доказаны.

\section{5. Доказательство теоремы 1: оценки снизу}

При получении оценок снизу, не ограничивая общности, будем предполагать, что $\omega=n$, т. е. $\frac{s_{1}}{m_{1}}=\ldots=\frac{s_{n}}{m_{n}}$.

Легко видеть, что для каждого оператора $T_{a} \in \mathfrak{H}_{\varepsilon \vartheta}^{\tau \mathrm{m}}\left[v ; \mathrm{K}_{\mathrm{f}}, \mathrm{L}\right]$, любых операторов информации $\mathcal{I}_{\Lambda}=\left\{\mathfrak{d}_{\xi} \mid \xi \in \Lambda\right\}\left(\Lambda \subset \mathbb{Z}^{\mathrm{m}}: \# \Lambda=N\right)$ и $\mathcal{J}_{\mathrm{M}}=\left\{\mathfrak{g}_{\xi, \zeta} \mid(\xi, \zeta) \in \mathrm{M}\right\}\left(\mathrm{M} \subset \mathbb{Z}^{\mathrm{m}} \times \mathbb{Z}^{\mathrm{m}}: \# \mathrm{M}<\infty\right)$ и любого метода $\Upsilon$ верна оценка

$$
\sup \left\{\left\|T_{a} f\left|L_{r} \|\right| f \in \mathrm{F}_{p q}^{s \mathrm{~m}}: \widehat{f}(\xi)=0, \xi \in \Lambda\right\} \leq \sup \left\{\left\|T_{a} f-\Upsilon\left(\mathcal{I}_{\Lambda}(f), \mathcal{J}_{\mathrm{M}}\left(T_{a}\right)\right)\left|L_{r} \|\right| f \in \mathrm{F}_{p q}^{s \mathrm{~m}}\right\} .\right.\right.
$$

Поэтому

$$
\begin{gathered}
\inf \left\{\sup \left\{\left\|T_{a} f\left|L_{r} \|\right| f \in \mathrm{F}_{p q}^{s \mathrm{~m}}: \widehat{f}(\xi)=0, \xi \in \Lambda\right\} \mid \Lambda \subset \mathbb{Z}^{m}: \# \Lambda \leq N\right\}\right. \\
\leq \mathfrak{S}\left(\mathfrak{H}_{\varepsilon \vartheta}^{\tau \mathrm{m}}\left[v ; \mathrm{K}_{\mathrm{f}}, \mathrm{L}\right], \mathrm{F}_{p q}^{s \mathrm{~m}} ; \mathfrak{I}_{N}, \mathfrak{J} ; L_{r}\right) .
\end{gathered}
$$

Дальнейшие оценки снизу будут получены именно для этого оператора $I^{(\tau)}:=T_{a(\tau)}$.

Сначала рассмотрим случай а) теоремы 1 . Итак, пусть $2 \leq p, q \leq \infty, r \leq p$.

Предположим, что $\Lambda-$ любое множество из $\mathbb{Z}^{m}$ с $\# \Lambda \leq N$. Выберем $u>0$ так, чтобы $2 \# \Lambda \leq \# \Lambda_{+}(u)$ и $\# \Lambda_{+}(u) \asymp N$, т. е. $N \asymp 2^{u} u^{n-1}$.

Тогда по теореме 2 найдется тригонометрический полином $t_{\Lambda, u} \in \mathrm{T}\left(\Lambda_{+}(u)\right)$ такой, что $\left\|t_{\Lambda, u} \mid L_{2}\right\|>c(\mathrm{~m})$ и $\widehat{t}_{\Lambda, u}(\xi)=0$ для всех $\xi \in \Lambda$, причем для нормы $\left\|t_{\Lambda, u} \mid F_{p q}^{s \mathrm{~m}}\right\|$ справедлива оценка (3.3). 
Определим полином $g(x):=I^{(-\tau)} t_{\Lambda, u}(x)$, тогда, очевидно,

$$
h:=\frac{g}{\left\|g \mid F_{p q}^{s \mathrm{~m}}\right\|} \in \mathrm{F}_{p q}^{s \mathrm{~m}}, \quad \widehat{h}(\xi)=0, \quad \xi \in \Lambda,
$$

кроме того, с учетом свойств оператора лифтинга (см. разд. 3) и оценки (3.3) находим

$$
\left\|I^{(\tau)} h\left|L_{r}\left\|=\frac{\left\|t_{\Lambda, u} \mid L_{r}\right\|}{\left\|g \mid F_{p q}^{s \mathrm{~m}}\right\|} \asymp \frac{\left\|t_{\Lambda, u} \mid L_{r}\right\|}{\left\|t_{\Lambda, u} \mid F_{p q}^{s-\tau \mathrm{m}}\right\|} \gg\right\| t_{\Lambda, u}\right| L_{r}\right\| 2^{-\sigma u} u^{(n-1)(1 / 2-1 / q)} .
$$

Если $r \geq 2$, то (поскольку $\left\|\cdot\left|L_{r}\|\geq\| \cdot\right| L_{2}\right\|$ ) отсюда следует, что

$$
\left\|I^{(\tau)} h \mid L_{r}\right\| \gg c(\mathrm{~m}) 2^{-\sigma u} u^{(n-1)(1 / 2-1 / q)} .
$$

Если же $r<2$, то, используя неравенство $\left\|t_{\Lambda, u}\left|L_{2}\|\leq\| t_{\Lambda, u}\right| L_{1}\right\|^{1 / 3}\left\|t_{\Lambda, u} \mid L_{4}\right\|^{2 / 3}$, вытекающее из неравенства Гёльдера, и оценку $\left\|t_{\Lambda, u} \mid L_{4}\right\| \ll 1$, которая доказывается вполне аналогично оценке $\left\|g_{2} \mid L_{4}\right\| \ll 1$ в работе [9, с. 276], снова отсюда получим (с учетом неравенства $\left\|\cdot \mid L_{r}\right\| \geq$ $\left.\left\|\cdot \mid L_{1}\right\|\right)$ оценку типа (5.2).

Таким образом из соотношений (5.1) и (5.2) вытекает требуемая оценка снизу (2.3), если принять во внимание замечание 1.

Теперь перейдем к рассмотрению случая b). Пусть $1 \leq q<2 \leq p \leq \infty, r \leq p$.

Сперва докажем оценку (2.4). Рассуждения п. 3 (там при получении оценки (5.2) нигде не использовалось условие $q \geq 2$ ) дают оценку

$$
\mathfrak{S}\left(\mathfrak{H}_{\varepsilon \vartheta}^{\tau \mathrm{m}}\left[v ; \mathrm{K}_{\mathrm{f}}, \mathrm{L}\right], \mathrm{F}_{p q}^{s \mathrm{~m}} ; \mathfrak{I}_{N}, \mathfrak{J} ; L_{r}\right) \gg 2^{-\sigma u} u^{(n-1)(1 / 2-1 / q)},
$$

которая в силу замечания 1 является искомой оценкой (2.4).

Рассмотрим случай е). Оценка (2.10) следует из оценок (2.3) и (2.4). В самом деле, в силу $(2.3),(2.4)$ и вложения $\mathrm{F}_{p q}^{s \mathrm{~m}} \supset \mathrm{F}_{2 q}^{s \mathrm{~m}}$ (которое вытекает из неравенства $\left\|\cdot\left|L_{p}\|\leq\| \cdot\right| L_{2}\right\|$ при $p<2$ ) имеем (принимая во внимание замечание 1 )

$$
\begin{aligned}
& \mathfrak{S}\left(\mathfrak{H}_{\varepsilon \vartheta}^{\tau \mathrm{m}}\left[v ; \mathrm{K}_{\mathrm{f}}, \mathrm{L}\right], \mathrm{F}_{p q}^{s \mathrm{~m}} ; \mathfrak{I}_{N}, \mathfrak{J} ; L_{r}\right) \\
& \geq \mathfrak{S}\left(\mathfrak{H}_{\varepsilon \vartheta}^{\tau \mathrm{m}}\left[v ; \mathrm{K}_{\mathrm{f}}, \mathrm{L}\right], \mathrm{F}_{2 q}^{s \mathrm{~m}} ; \mathfrak{I}_{N}, \mathfrak{J} ; L_{r}\right) \gg \varphi_{N}\left(\mathrm{~F}_{2 q}^{s \mathrm{~m}}, L_{r}\right)\left(\log ^{\omega-1} N\right)^{-(1 / q-1 / 2)_{+}} \\
& =\left(\frac{\log ^{\omega-1} N}{N}\right)^{\sigma}\left(\log ^{\omega-1} N\right)^{(1 / 2-1 / q)_{+}-(1 / q-1 / 2)_{+}}=\left(\frac{\log ^{\omega-1} N}{N}\right)^{\sigma}\left(\log ^{\omega-1} N\right)^{(1 / 2-1 / q)} \text {. }
\end{aligned}
$$

При получении оценок снизу в соотношениях $(2.5),(2.7),(2.9)$ нам понадобятся оценки линейных поперечников классов $\mathrm{F}_{p q}^{s-\tau \mathrm{m}}$ в пространстве $L_{r}$ из [15]. Напомним, что $N$-м линейным поперечником множества $\mathrm{F}$ банахова пространства $X$ (с нормой $\|\cdot \mid X\|)$ называется величина

$$
\lambda_{N}(\mathrm{~F}, X):=\inf _{A \in \mathcal{L}(X): \operatorname{rank}(A) \leq N} \sup _{f \in \mathrm{F}}\|f-A f \mid X\|,
$$

здесь $\mathcal{L}(X)$ - пространство линейных непрерывных операторов $A: X \rightarrow X$. Линейные поперечники были введены В. М. Тихомировым в 1960 г.

Нетрудно видеть, что верна оценка

$$
\mathfrak{S}^{\prime}\left(\mathfrak{H}_{\varepsilon \vartheta}^{\tau \mathrm{m}}\left[v ; \mathrm{K}_{\mathrm{f}}, \mathrm{L}\right], \mathrm{F}_{p q}^{s \mathrm{~m}} ; \mathfrak{I}_{N}, \mathfrak{J} ; L_{r}\right) \gg \lambda_{N}\left(\mathrm{~F}_{p q}^{s-\tau \mathrm{m}}, L_{r}\right) .
$$

Но в условиях пп. b)-d) теоремы 1 в силу теоремы 2 из [15] (см. там пп. (i), (ii), (v) соответственно) имеет место порядковое равенство

$$
\lambda_{N}\left(\mathrm{~F}_{p q}^{s-\tau \mathrm{m}}, L_{r}\right) \asymp \varphi_{N}\left(\mathrm{~F}_{p q}^{s-\tau \mathrm{m}}, L_{r}\right),
$$

которое завершает доказательство оценок снизу в соотношениях $(2.5),(2.7),(2.9)$. 
Таким образом, все оценки снизу в теореме 1 доказаны полностью.

З а м е ч а н и е 3 . В условиях пункта е)

- для классов Лизоркина - Трибеля при $1<p<2 \leq q \leq \infty$ (снова с учетом замечания 1$)$, очевидно, имеем

$$
\mathfrak{S}\left(\mathfrak{H}_{\varepsilon \vartheta}^{\tau \mathrm{m}}\left[v ; \mathrm{K}_{\mathrm{f}}, \mathrm{L}\right], \mathrm{L}_{p q}^{s \mathrm{~m}} ; \mathfrak{I}_{N}, \mathfrak{J} ; L_{r}\right) \asymp \varphi_{N}\left(\mathrm{~L}_{p q}^{s \mathrm{~m}}, L_{r}\right) ;
$$

- для классов Лизоркина - Трибеля при остальных (допустимых) соотношениях между $p$ и $q$, а также для классов Никольского - Бесова при любых (допустимых) соотношениях между $p$ и $q$ оценка сверху (2.2) и оценка снизу (2.10) совпадают в степенной шкале, но между ними имеется "логарифмический люфт".

\section{6. Заключительные замечания и комментарии ${ }^{3}$}

Как было отмечено во введении, исследованию разнообразных задач (оптимального) восстановления (значений) операторов посвящена обширная литература. История и различные аспекты этой проблематики отражены, в частности, в монографиях [3;6;16-19] и обзорах [1;2;5] (см. также библиографию в обсуждаемых ниже статьях).

Здесь в связи с теоремой 1 обсудим лишь результаты ряда работ, в которых изучаются различные аспекты задач (оптимального) восстановления операторов на классах периодических функций одной и нескольких переменных по спектральной информации или близкие задачи.

Прежде всего отметим работу [20], в которой изучается (в наших обозначениях) задача (1.1), где рассматриваются гильбертово пространство $Y=L_{2}(M)$ (с окружностью $\mathbb{T}$, кругом $D$ в комплексной плоскости или многомерной сферой $\mathbb{S}^{d}$ в качестве $\left.M\right)$ с полной ортонормированной системой $\left\{\psi_{l}\right\}_{l \in \mathcal{N}}$ (в случае окружности берется тригонометрическая система $\left\{e^{2 \pi i l x}\right\}_{l \in \mathbb{Z}}$, в случае круга $-\left\{z^{n}\right\}_{n \in \mathbb{N}_{0}}$, а в случае сферы - система сферических гармоник), единичный шар $\mathrm{F}$ некоторого подпространства $X$ из $L_{2}(M)$ (типичные примеры в случае окружности - классы Соболева $W_{2}^{s}(\mathbb{T})$ и Харди $H_{2}^{s}(\mathbb{T})$, Бергмана - Соболева $A_{2}^{s, \beta}(\mathbb{T})$, в случае круга - Соболева - Харди $H_{2}^{s}(D)$, Бергмана - Соболева $A_{2}^{s, \beta}(D)$, а в случае сферы - классы типа Соболева $W_{2}^{\beta}\left(\mathbb{S}^{d}\right)$, определяемые ограничениями в метрике $L_{2}\left(\mathbb{S}^{d}\right)$ на действие (дробной) степени оператора Лапласа $(-\Delta)^{\beta / 2}$ ) и оператор $T: X \rightarrow L_{2}(M)$ типа мультипликатора (типичные примеры в случае окружности и круга - тождественный оператор и (промежуточная) производная $d^{k} / d x^{k}$, а в случае сферы - тождественный оператор и (“промежуточная" дробная) степень лапласиана $(-\Delta)^{\gamma / 2}$ ). В качестве (оператора) информации рассматривается (полный или конечный) набор коэффициентов Фурье $\left\{f_{l}\right\}_{l \in \mathrm{N}}$ функции $f \in \mathrm{F}$ по системе $\left\{\psi_{l}\right\}_{l \in \mathcal{N}}$ $(\mathrm{N} \subset \mathcal{N})$, заданный с некоторой погрешностью (в той или иной метрике); таким образом, если погрешность ненулевая, то оператор информации $\mathcal{I}$ является, вообще говоря, многозначным, и тогда задача (1.1) принимает вид

$$
\begin{gathered}
\mathfrak{S}(T, \mathrm{~F} ; \mathcal{I} ; Y):=\inf \{\mathfrak{R}(T, \mathrm{~F} ; \mathcal{I}, \Upsilon ; Y) \mid \Upsilon\} \\
\mathfrak{R}(T, \mathrm{~F} ; \mathcal{I}, \Upsilon ; Y):=\sup \{\|T f-\Upsilon(y)|Y \|| y \in \mathcal{I}(f), f \in \mathrm{F}\}
\end{gathered}
$$

В [20] применяется подход, основанный на стандартных принципах выпуклой оптимизации, и при некоторых ограничениях на оператор $T$ (все перечисленные выше примеры операторов им удовлетворяют) дается точное решение задачи (1.1): вычисляется точное значение величины (1.1) и выписываются явные выражения для оптимальных методов. Подчеркнем, что в этом подходе ключевую роль играет гильбертова структура пространства $Y$ и класса F. Дальнейшее его развитие и применение к решению других задач оптимального восстановления можно проследить, в частности, по работам [21-23]. Отметим, что в [23] развивается метод, дающий точное решение задач оптимального восстановления линейных операторов по

\footnotetext{
${ }^{3}$ По согласованию с редакцией здесь приведен краткий обзор исследований, близких по тематике к данной работе.
} 
(неточной) спектральной информации в ряде случаев неевклидовых метрик (т. е. при отказе от гильбертовости); подробности см. [23, §7].

В серии работ [24-28] проведено весьма полное исследование задачи оптимизации по сложности приближенного решения уравнений Фредгольма второго рода с гладкими ядрами. Вопрос о (точном порядке) сложности приближенного решения (классов) операторных уравнений относится к теории информационной сложности (information - based complexity) и состоит в определении (точного порядка) количества элементарных (в том или ином смысле) операций, потребного для построения приближенного решения таких уравнений с наперед заданной точностью (в той или иной метрике). Мы не будем входить в подробности, лишь сошлемся на монографии [3;17-19], первая из которых фактически положила начало этой теории, а три последние подводят некоторый промежуточный итог в ее развитии.

Здесь же для нас важно отметить в работах [24-28] ряд важных обстоятельств в связи с рассмотренной выше задачей оптимального восстановления (1.5).

Пусть $C\left(\mathbb{T}^{2}\right)$ - пространство непрерывных на торе $\mathbb{T}^{m}$ функций. Обозначим через $A W_{2}^{s}(\mathbb{T})$ $(A>0)$ множество всех функций $f \in W_{2}^{s}(\mathbb{T})$ (см. пример 1 из разд. 1 выше) таких, что $\left\|f\left|L_{2}\left(\mathbb{T}^{2}\right)\|+\| f^{(s)}\right| L_{2}\left(\mathbb{T}^{2}\right)\right\| \leq A$, через $B W_{2}^{s, s}\left(\mathbb{T}^{2}\right)$ - множество $(B>0)$

$$
\left\{h \in C\left(\mathbb{T}^{2}\right)\left|\partial^{(\alpha, \beta)} h \in C\left(\mathbb{T}^{2}\right), 0 \leq \alpha, \beta \leq s, \sum_{0 \leq \alpha, \beta \leq s}\left\|\partial^{(\alpha, \beta)} h \mid L_{2}\left(\mathbb{T}^{2}\right)\right\| \leq B\right\}\right.
$$

и через $\mathfrak{H}^{s}(B, C)(C>0)$ - класс всех интегральных операторов

$$
T_{h}: L_{2}(\mathbb{T}) \rightarrow L_{2}(\mathbb{T}): f \mapsto T_{h} f:=\int_{\mathbb{T}} h(\cdot, y) f(y) d y
$$

с ядрами $h \in B W_{2}^{s, s}\left(\mathbb{T}^{2}\right)$ таких, что оператор $I d-T_{h}: L_{2}(\mathbb{T}) \rightarrow L_{2}(\mathbb{T})$ обратим (здесь $I d-$ тождественный оператор), причем

$$
\left\|\left(I d-T_{h}\right)^{-1} \mid L_{2}(\mathbb{T}) \rightarrow L_{2}(\mathbb{T})\right\| \leq C .
$$

В [24-26;28] (в последней работе рассматривается многомерный случай) изучается задача (оценки сложности) приближенного восстановления решения уравнения Фредгольма второго рода (в наших обозначениях)

$$
g=T_{h} g+f
$$

с $T_{h} \in \mathfrak{H}^{s}(B, C)$ и $f \in A W_{2}^{s}(\mathbb{T})$ фактически как задача восстановления значений $\widetilde{T}_{h} f$ операторов $\widetilde{T}_{h}$ из класса

$$
\mathfrak{H}^{-s}(B, C):=\left\{\widetilde{T}_{h}=\left(I d-T_{h}\right)^{-1} \mid T_{h} \in \mathfrak{H}^{s}(B, C)\right\}
$$

на функциональном классе $A W_{2}^{s}(\mathbb{T})$ по информации об $f$ в виде набора тригонометрических коэффициентов Фурье $\{\widehat{f}(l):|l| \leq N\}$ и (неполной!) информации об операторе $\widetilde{T}_{h}$ в виде набора тригонометрических коэффициентов Фурье $\left\{\widehat{h}(l, k):(k, l) \in \Xi_{N}\right\}$ ядра $h$ исходного оператора $T_{h}$, где $\Xi_{N}$ - подходящим (оптимальным в соответствующем смысле) образом выбранный гиперболический крест из $\mathbb{Z}^{2}$. Кроме того, в этих работах для получения требуемых оценок снизу привлекаются поперечники соответствующих функциональных компактов (например, в [26] для этих целей используются поперечники Александрова, в [27;28] - поперечники Гельфанда). Между прочим, здесь же отметим, что оценки снизу, доказанные в разд.5 с использованием теремы 2 из разд.3, фактически дают оценки снизу для соответствующих поперечников Гельфанда классов $\mathrm{F}_{p q}^{s \mathrm{~m}}$.

\section{СПИСОК ЛИТЕРАТУРЫ}

1. Micchelli C.A., Rivlin T.J. A survey of optimal recovery // Optimal Estimation in Approximation Theory /eds. C.A. Micchelli, T.J. Rivlin. N Y etc.: Plenum, 1977. P. 1-54.

doi: 10.1007/978-1-4684-2388-4_1. 
2. Micchelli C.A., Rivlin T.J. Lectures on optimal recovery // Numerical Analysis Lancaster / ed. P.R. Turner. Berlin: Springer-Verlag, 1984. P. 21-93. (Lecture Notes Math.; vol. 1129). doi: $10.1007 /$ BFb0075157.

3. Трауб Дж., Вожьняковский Х. Общая теория оптимальных алгоритмов. М.: Мир, 1983. 382 с.

4. Арестов В.В. Наилучшее восстановление операторов и родственные задачи // Тр. МИАН СССР. 1989. T. 189. C. $3-20$.

5. Арестов В.В. Приближение неограниченных операторов ограниченными и родственные экстремальные задачи // Успехи мат. наук. 1996. Т. 51, вып. 6. С. 89-124.

6. Женсыкбаев А.А. Проблемы восстановления операторов. М.; Ижевск: Регулярная и хаотическая динамика, 2003. $411 \mathrm{c}$.

7. Dinh Dung, Temlyakov V.N., Ullrich T. Hyperbolic cross approximation. Basel: Birkhäuser Springer, 2018. 218 p. doi: 10.1007/978-3-319-92240-9.

8. Базарханов Д.Б. Приближение всплесками и поперечники Фурье классов периодических функций многих переменных. I // Тр. МИАН. 2010. Т. 269. С. 8-30.

9. Базарханов Д.Б. Приближение всплесками и поперечники Фурье классов периодических функций многих переменных. II // Analysis Math. 2012. Vol. 38, no. 4. C. 249-289. doi: 10.1007/s10476-012-0401-3.

10. Темляков В.Н. Оценки асимптотических характеристик классов функций с ограниченной смешанной производной или разностью // Тр. МИАН СССР. 1989. Т. 189. С. 138-168.

11. Ruzhansky M., Turunen V. Pseudo-differential operators and symmetries: background analysis and advanced topics. Basel: Birkhäuser Springer, 2009. 709 p. doi: 10.1007/978-3-7643-8514-9 .

12. Никольский С. М. Приближение функций многих переменных и теоремы вложения. 2-е изд. Москва: Наука, 1977. 456 с.

13. Трибель Х. Теория функциональных пространств. М.: Мир, 1986. 448 р.

14. Schmeisser H. J., Triebel H. Topics in Fourier analysis and function spaces. Chichester: J. Wiley \& Sons, 1987. $300 \mathrm{p}$.

15. Bazarkhanov D.B. Estimates for the widths of classes of periodic functions of several variables. I // Eurasian Math. J. 2010. Vol. 1, no. 3. C. 11-26.

16. Теоретические основы и конструирование численных алгоритмов для задач математической физики / ред. К.И. Бабенко. М.: Наука, 1979. 295 с.

17. Novak E., Wozniakowski H. Tractability of multivariate problems. Vol. I: Linear information. Zurich: EMS Tracts Math., 2008.

18. Novak E., Wozniakowski H. Tractability of multivariate problems. Vol. II: Standard information for functionals. Zurich: EMS Tracts Math., 2010.

19. Novak E., Wozniakowski H. Tractability of multivariate problems. Vol. III: Standard information for operators. Zurich: EMS Tracts Math., 2012.

20. Магарил - Ильяев Г. Г., Осипенко К. Ю. Оптимальное восстановление функций и их производных по коэффициентам Фурье, заданным с погрешностью // Мат. сб. 2002. Т. 193, № 3 С. 79-100.

21. Выск Н.Д., Осипенко К. Ю. Оптимальное восстановление решения волнового уравнения по неточным начальным данным // Мат. заметки. 2007. Т. 81, № 6 С. 803-815.

22. Магарил - Ильяев Г. Г., Осипенко К. Ю. О наилучшем гармоническом синтезе периодических функций // Фунд. и прикладная математика. 2013. Т. 18, № 5 С. 155-174.

23. Осипенко К. Ю. Оптимальное восстановление линейных операторов в неевклидовых метриках // Мат. сб. 2014. Т. 205, № 10 C. 77-106.

24. Переверзев С. В. О сложности задачи нахождения решений уравнений Фредгольма II рода с гладкими ядрами. I // Укр. мат. журн. 1988. Т. 40, № 1 С. 84-91.

25. Переверзев С. В. О сложности задачи нахождения решений уравнений Фредгольма II рода с гладкими ядрами. II // Укр. мат. журн. 1989. Т. 41, № 2 С. 189-193.

26. Переверзев С. В. Гиперболический крест и сложность приближенного решения уравнений Фредгольма II рода с дифференцируемыми ядрами. // Сиб. мат. журн. 1991. Т. 32, № 1. С. 107-115.

27. Heinrich S. Complexity of integral equations and relation to $s$-numbers // J. Complexity. 1993. Vol. 9, no. 1. P. 141-153. doi: 10.1006/jcom.1993.1010.

28. Frank K., Heinrich S., Pereverzev S. V. Information complexity of multivariate Fredholm integral equations in Sobolev classes // J. Complexity. 1996. Vol. 12, no. 1. P. 17-34. doi: 10.1006/jcom.1996.0004 . 
Поступила 9.08.2019

После доработки 18.11.2019

Принята к публикации 25.11.2019

Базарханов Даурен Болысбекович

канд. физ.-мат. наук, профессор

главный науч. сотрудник, зав. отделом теории функций

Институт математики и математического моделирования

г. Алматы

e-mail: dauren.mirza@gmail.com

\section{REFERENCES}

1. Micchelli C.A., Rivlin T.J. A survey of optimal recovery. In: Optimal estimation in approximation theory. N.Y. etc.: Plenum Press, 1977, pp. 1-54. doi: 10.1007/978-1-4684-2388-4_1.

2. Micchelli C.A., Rivlin T.J. Lectures on optimal recovery. In: Turner P.R. (ed.), Numerical Analysis Lancaster (1984), Lecture Notes in Mathematics, vol. 1129, Berlin: Springer-Verlag, pp. 21-93. doi: $10.1007 / \mathrm{BFb} 0075157$.

3. Traub J., Wozniakowski H. A general theory of optimal algorithms. ACM Monograph Ser., N Y etc.: Acad. Press, 1980, 341 p. ISBN: 0126976503. Translated to Russian under the title Obshchaya teoriya optimal'nykh algoritmov. Moscow: Mir Publ., 1983, 382 p.

4. Arestov V.V. Optimal recovery of operators and related problems. Proc. Steklov Inst. Math., 1990, no. 4, pp. $1-20$.

5. Arestov V.V. Approximation of unbounded operators by bounded operators and related extremal problems. Russ. Math. Surv., 1996, vol. 51, no. 6, pp. 1093-1126. doi: 10.1070/RM1996v051n06ABEH003001.

6. Zhensykbaev A.A. Problemy vosstanovleniya operatorov (Operator recovery problems). Moscow; Izhevsk: RKhD Publ., 2003, 411 p. ISBN: 5-93972-268-7.

7. Dinh Dung, Temlyakov V.N., Ullrich T. Hyperbolic cross approximation. Basel: Birkhäuser Springer, 2018, 218 p. doi: 10.1007/978-3-319-92240-9 .

8. Bazarkhanov D.B. Wavelet approximation and Fourier widths of classes of periodic functions of several variables. I. Proc. Steklov Inst. Math., 2010, vol. 269, pp. 2-24. doi: 10.1134/S0081543810020021.

9. Bazarkhanov D.B. Wavelet approximation and Fourier widths of classes of periodic functions of several variables. II. Analysis math., 2012, vol. 38, no. 4, pp. 249-289. doi: 10.1007/s10476-012-0401-3.

10. Temlyakov V.N. Estimates for the asymptotic characteristics of classes of functions with bounded mixed derivative or difference. Proc. Steklov Inst. Math., 1990, vol. 189, pp. 161-197.

11. Ruzhansky M., Turunen V. Pseudo-differential operators and symmetries: background analysis and advanced topics. Basel: Birkhäuser Springer, 2009, 709 p. doi: 10.1007/978-3-7643-8514-9 .

12. Nikol'skii S.M. Approximation of Functions of Several Variables and Embedding Theorems. Berlin; N Y: Springer-Verlag, 1975, 420 p. doi: 10.1007/978-3-642-65711-5 . Original Russian text (2nd ed.) published in Nikol'skii S.M. Priblizhenie funktsii mnogikh peremennykh i teoremy vlozheniya. Moscow: Nauka Publ., 1977, $456 \mathrm{p}$.

13. Triebel H. Theory of function spaces. Basel: Birkhäuser, 1983. ISBN: 3764313811 . Translated to Russian under the title Teoriya funktsional'nykh prostranstv. Moscow: Mir Publ., 1986.

14. Schmeisser H.J., Triebel H. Topics in Fourier analysis and function spaces. Chichester: J. Wiley \& Sons, 1987, 300 p. ISBN: 0-471-90895-9.

15. Bazarkhanov D. Estimates for the widths of classes of periodic functions of several variables. I. Eurasian Math. J., 2010, vol. 1, no. 3, pp. 11-26.

16. Babenko K.I. (eds) Teoreticheskie osnovy $i$ konstruirovanie chislennykh algoritmov dlya zadach matematicheskoi fiziki [Theoretical principles and construction of numerical algorithms of mathematical physics]. Moscow: Nauka Publ., 1979, 295 p.

17. Novak E., Wozniakowski H. Tractability of multivariate problems. Vol. I: Linear information. EMS Tracts in Mathematics, Zurich: EMS, 2008. ISBN: 978-3-03719-026-5.

18. Novak E., Wozniakowski H. Tractability of multivariate problems. Volume II: Standard information for functionals. EMS Tracts in Mathematics, Zurich: EMS, 2010. ISBN: 9783037190845.

19. Novak E., Wozniakowski H. Tractability of multivariate problems. Volume III: Standard information for operators. EMS Tracts in Mathematics, Zurich: EMS, 2012. ISBN: 9783037191163. 
20. Magaril-Il'yaev G.G., Osipenko K.Yu. Optimal recovery of functions and their derivatives from Fourier coefficients prescribed with an error. Sb. Math., 2002, vol. 193, no. 3, pp. 387-407. doi: 10.1070/SM2002v193n03ABEH000637.

21. Vysk N.D., Osipenko K.Yu. Optimal Reconstruction of the Solution of the Wave Equation from Inaccurate Initial Data. Math. Notes, 2007, vol. 81, no. 6, pp. 723-733. doi: 10.1134/S0001434607050203.

22. Magaril-Il'yaev G.G., Osipenko K.Yu. On best harmonic synthesis of periodic functions. J. Math. Sci., 2015, vol. 209, no. 1, pp. 115-129. doi: 10.1007/s10958-015-2489-z .

23. Osipenko K.Yu. Optimal recovery of linear operators in non-Euclidean metrics. Sb. Math., 2014, vol. 205, no. 10, pp. 1442-1472. doi: 10.1070/SM2014v205n10ABEH004425.

24. Pereverzev S.V. Complexity of the problem of finding the solutions of fredholm equations of the second kind with smooth kernels. I. Ukr. Math. J., 1988, vol. 40, no. 1, pp. 71-76. doi: 10.1007/BF01056451 .

25. Pereverzev S.V. Complexity of the problem of finding the solutions of Fredholm equations of the second kind with smooth kernels. II. Ukr. Math. J., 1989, vol. 41, no. 2, pp. 169-173. doi: 10.1007/BF01060382.

26. Pereverzev S.V. Hyperbolic cross and the complexity of the approximate solution of Fredholm integral equations of the second kind with differentiable kernels. Sib. Math. J., 1991, vol. 32, no. 1, pp. 85-92. doi: $10.1007 /$ BF00970164.

27. Heinrich S. Complexity of integral equations and relations to s-numbers. J. Complexity, 1993, vol. 9, no. 1, pp. 141-153. doi: 10.1006/jcom.1993.1010.

28. Frank K., Heinrich S., Pereverzev S.V. Information complexity of multivariate Fredholm integral equations in Sobolev classes. J. Complexity, 1996, vol. 12, no. 1, pp. 17-34. doi: 10.1006/jcom.1996.0004.

Received August 9, 2019

Revised November 8, 2019

Accepted November 25, 2019

Funding Agency: This work was supported by the Ministry of Education and Science of the Republic of Kazakhstan (grant no. AP05133257).

Dauren B. Bazarkhanov, Cand. Sci. (Phys.-Math.), Prof., Institute of Mathematics and Math Modeling, Almaty, 050010 Kazakhstan, e-mail: dauren.mirza@gmail.com .

Cite this article as: D. B. Bazarkhanov. Linear recovery of pseudodifferential operators on classes of smooth functions on an m-dimensional torus. II, Trudy Instituta Matematiki i Mekhaniki URO $R A N, 2019$, vol. 25 , no. 4 , pp. 15-30. 